\title{
A CLASS OF STRONGLY STABLE OPERATOR APPROXIMATIONS
}

\author{
MARIO AHUES ${ }^{1}$
}

(Received 11 October 1985; revised 20 May 1986)

\begin{abstract}
We show the strongly stable convergence of some non-collectively-compact approximations of compact operators. Special attention is devoted to Anselone's singularity subtraction discretization of certain singular integral operators. Numerical experiments are provided.
\end{abstract}

\section{Introduction}

It is well known (cf. [3]) that uniform and collectively-compact approximations of compact operators in infinite dimensional complex Banach spaces are strongly stable in a neighborhood of each nonzero eigenvalue. This means, roughly speaking, that nonzero eigenvalues are approximated with preservation of their algebraic multiplicity and maximal invariant subspaces associated with them are approximated with convergence in gap. The notion of strongly stable convergence was introduced by Chatelin in [3].

However, for singular compact integral operators, Anselone's collectively-compact theory (in [1]) does not cover some commonly used discretizations such as singularity subtraction, proposed by Anselone himself in [2].

In this work we prove that:

If $T$ is a compact linear operator in an infinite dimensional complex Banach space $X$ and $\left(T_{n}\right)$ is a sequence of linear bounded operators in $X$ (which need not

\footnotetext{
${ }^{1}$ Departamento de Matemáticas, Facultad de Ciencias Físicas y Matemáticas, Universidad de Chile, Casilla 170/3 Santiago 3

(c) Copyright Australian Mathematical Society 1987, Serial-fee code 0334-2700/87
} 
be compact) such that

C1. $\forall x \in X \lim _{n \rightarrow \infty} T_{n} x=T x$,

C2. $\lim _{n \rightarrow \infty}\left\|\left(T_{n}-T\right) T_{n}\right\|=0$,

then $\left(T_{n}\right)$ is a strongly stable approximation to $T$ in a neighborhood of each nonzero eigenvalue of $T$.

Conditions $\mathrm{C} 1$ and $\mathrm{C} 2$ are satisfied by uniform and collectively-compact discretizations but they are also satisfied by norm perturbations of collectively compact approximations $T_{n}$ of a compact $T$, namely, $T_{n}=K_{n}+B_{n}$ where $B_{n}$ converges in norm to 0 and $K_{n}$ is a collectively compact approximation to $T$.

A singularity subtraction example is discussed in Section 4.

\section{Mathematical background}

Let $X$ be a complex infinite dimensional Banach space. \|| || will denote both the norm of $X$ and that of the algebra $\mathscr{L}(X)$, the space of linear bounded operators defined in $X .1$ stands for the identity in $X$ and $z$ denotes $z 1$ for any complex number $z$.

We state in this section a set of lemmas which will be needed in the proof of our main theorem, to which is devoted the next section. Lemmas 1 and 6 are well-known results, so proofs will be omitted.

Lemma 1. If $\left(A_{n}\right) \subseteq \mathscr{L}(X), A \in \mathscr{L}(X)$ and $\forall x \in X A_{n} x \rightarrow A x$, then there is a constant $C>0$ and an integer $n_{0}$ such that $\sup _{n}>n_{0}\left\|A_{n}\right\| \leqslant C$.

LeMmA 2. If $\left(A_{n}\right) \subseteq \mathscr{L}(X),\left(B_{n}\right) \subseteq \mathscr{L}(X), \quad A \in \mathscr{L}(X), B \in \mathscr{L}(X)$ and $\forall x \in X A_{n} x \rightarrow A x$ and $B_{n} x \rightarrow B x$, then $\forall x \in X A_{n} B_{n} x \rightarrow A B x$.

LeMMA 3. If $\left(A_{n}\right) \subseteq \mathscr{L}(X), \quad A \in \mathscr{L}(X), \quad K \in \mathscr{L}(X)$ is compact and $\forall x \in X A_{n} x \rightarrow A x$, then $\left\|\left(A_{n}-A\right) K\right\| \rightarrow 0$.

LEMMA 4. If $\Gamma$ is a compact subset of the complex plane, $z \in \Gamma \rightarrow U(z) \in \mathscr{L}(X)$ is a continuous function in $\Gamma,\left(A_{n}\right) \subseteq \mathscr{L}(X), A \in \mathscr{L}(X)$ and $\forall x \in X, A_{n} x \rightarrow A x$, then $\forall x \in X, \sup _{z \in \Gamma}\left\|\left(A_{n}-A\right) U(z) x\right\| \rightarrow 0$.

Let $T \in \mathscr{L}(X)$ be compact and $\left(T_{n}\right) \subseteq \mathscr{L}(X)$ such that

C1. $\forall x \in X, T_{n} x \rightarrow T x$,

C2. $\left\|\left(T_{n}-T\right) T_{n}\right\| \rightarrow 0$. 
Let $\lambda$ be a nonzero eigenvalue of $T$ isolated by a simple closed Jordan cuve $\Gamma$ not enclosing 0 and lying in the resolvent set $\rho(T)$ of $T$. For $z \in \Gamma$ we set $R(z)=(T-z)^{-1}$. The spectral projection associated with $\lambda$ is

$$
P=-\frac{1}{2 \pi i} \int_{\Gamma} R(z) d z \quad\left(i^{2}=-1\right)
$$

and it has finite rank, say $m$. The image space $M$ of $P$ is the (maximal) invariant subspace associated with $\lambda$ and has dimension $m$.

We formally set

$$
R_{n}(z)=\left(T_{n}-z\right)^{-1}, \quad P_{n}=-\frac{1}{2 \pi i} \int_{\Gamma} R_{n}(z) d z, \quad M_{n}=\text { Image space of } P_{n} .
$$

LEMMA 5. $z \in \Gamma \rightarrow R(z) \in \mathscr{L}(X)$ is continuous.

LEMMA 6. $T, P$ and, for $z \in \rho(T), R(z)$ commute one with each other.

LEMMA 7. Under conditions $\mathrm{C} 1$ and $\mathrm{C} 2$ there exists an integer $n_{0}$ such that, for $n>n_{0}, R_{n}(z)$ exists and belongs to $\mathscr{L}(X)$. Moreover

$$
\begin{aligned}
& \forall x \in X \forall z \in \Gamma, R_{n}(z) x \rightarrow R(z) x . \\
& \exists C_{\Gamma}>0 \text { such that } \sup _{\substack{z \in \Gamma \\
n>n_{0}}}\left\|R_{n}(z)\right\| \leqslant C_{\Gamma} .
\end{aligned}
$$

Proof. There exists $n_{0}$ and $\delta$ such that for $n>n_{0}$ :

$$
\forall z \in \Gamma \frac{1}{|z|}\left\|R(z)\left(T-T_{n}\right) T_{n}\right\| \leqslant \delta<1
$$

and

$$
r_{\sigma}\left(T-T_{n}\right)<|z|,
$$

where $r_{\sigma}$ denotes spectral radius. Then, for $n>n_{0}$,

$$
1-\frac{1}{z} R(z)\left(T-T_{n}\right) T_{n}=R(z)\left(1-\frac{1}{z}\left(T-T_{n}\right)\right)\left(T_{n}-z\right)
$$

is nonsingular with inverse in $\mathscr{L}(X)$, and the same holds for $1-\frac{1}{z}\left(T-T_{n}\right)$. Hence, for $n>n_{0}$ we have $z \in \rho\left(T_{n}\right)$ and

$$
\begin{aligned}
R_{n}(z) & =\left(1-\frac{1}{z} R(z)\left(T-T_{n}\right) T_{n}\right)^{-1} R(z)\left(1-\frac{1}{z}\left(T-T_{n}\right)\right) \\
& =\left(1+\sum_{k=1}^{\infty}\left(\frac{1}{z} R(z)\left(T-T_{n}\right) T_{n}\right)^{k}\right) R(z)\left(1-\frac{1}{z}\left(T-T_{n}\right)\right),
\end{aligned}
$$


so the constant $C_{\Gamma}$ in the conclusion of the lemma may be chosen as

$$
C_{\Gamma}=\frac{1}{1-\delta} \sup _{\substack{z \in \Gamma \\ n>n_{0}}}\left\|R(z)\left(1-\frac{1}{z}\left(T-T_{n}\right)\right)\right\|
$$

which is certainly finite.

Finally, for $z \in \Gamma$ and $n>n_{0}$ :

$$
R(z)-R_{n}(z)=R_{n}(z)\left(T_{n}-T\right) R(z),
$$

so $\forall x \in X, \forall z \in \Gamma, \forall n>n_{0}$

$$
\left\|\left(R(z)-R_{n}(z)\right) x\right\| \leqslant C_{\Gamma}\left\|\left(T_{n}-T\right) R(z) x\right\| \rightarrow 0 .
$$

LEMMA 8. Under conditions $\mathrm{C} 1$ and $\mathrm{C} 2$ there exists an integer $n_{0}$ such that for $n>n_{0}, P_{n}$ is well defined, belongs to $\mathscr{L}(X)$ and is a projection onto $M_{n}$. Moreover, $\forall x \in X, P_{n} x \rightarrow P x$.

PROOF. For the integer $n_{0}$ of Lemma 7 we have: $\forall x \in X, \forall n>n_{0}$,

$$
\begin{aligned}
\left\|\left(P_{n}-P\right) x\right\| & =\frac{1}{2 \pi}\left\|\int_{\Gamma}\left(R_{n}(z)-R(z)\right) x d z\right\| \\
& \leqslant C \sup _{z \in \Gamma}\left\|\left(T-T_{n}\right) R(z) x\right\| \rightarrow 0
\end{aligned}
$$

for some constant $C>0$.

\section{The main result.}

We first recall the notion of strongly stable approximation, keeping the notation of the preceding sections.

$\left(T_{n}\right)$ is a strongly stable approximation to $T$ around the eigenvalue $\lambda$ if the following conditions are satisfied, in addition to the pointwise convergence $\mathrm{Cl}$ :

SS1) For any Jordan curve $\Gamma$ isolating $\lambda$ and all $n$ large enough, $R_{n}(z)$ is defined for $z \in \Gamma$, belongs to $\mathscr{L}(X)$ and $\forall x \in X, R_{n}(z) x \rightarrow R(z) x$.

SS2) For all $n$ large enough, $\operatorname{dim} M_{n}=\operatorname{dim} M$.

We now state and prove the main result of the paper.

THEOREM. Under conditions $\mathrm{C} 1$ and $\mathrm{C} 2,\left(T_{n}\right)$ is a strongly stable approximation to $T$ around $\lambda$.

Proof. By Lemma 7, $\left(T_{n}\right)$ satisfies SS1. Hence, we only need to prove that there is an integer $n_{0}$ such that for $n>n_{0}$ :

$$
\operatorname{dim} M_{n}=\operatorname{dim} M \text {. }
$$


Let $\gamma\left(M, M_{n}\right)$ denote the gap between $M$ and $M_{n}$, then (cf. [3]):

$$
\gamma\left(M, M_{n}\right) \leqslant \max \left\{\left\|\left(P-P_{n}\right) P\right\|,\left\|\left(P-P_{n}\right) P_{n}\right\|\right\}
$$

and

$$
\gamma\left(M, M_{n}\right)<1 \text { implies } \operatorname{dim} M_{n}=\operatorname{dim} M,
$$

since $M$ is finite dimensional.

By Lemmas 3 and 8, since $P$ is of finite rank and hence compact,

$$
\left\|\left(P_{n}-P\right) P\right\| \rightarrow 0 .
$$

We shall prove that $\left\|\left(P_{n}-P\right) P_{n}\right\| \rightarrow 0$. Since $R_{n}(z)$ and $P_{n}$ commute:

$$
\left\|\left(P_{n}-P\right) P_{n}\right\|=\frac{1}{2 \pi}\left\|\int_{\Gamma} R(z)\left(T-T_{n}\right) R_{n}(z) P_{n} d z\right\| \leqslant C\left\|\left(T-T_{n}\right) P_{n}\right\|
$$

for some constant $C>0$ and $n$ large enough. It suffices now to prove that $\left\|\left(T-T_{n}\right) P_{n}\right\| \rightarrow 0$. We consider the decomposition

$$
\left(T-T_{n}\right) P_{n}=\left(T-T_{n}\right) P_{n} P+\left(T-T_{n}\right) P_{n}(1-P) \text {. }
$$

By Lemma 2: $\forall x \in X\left(T_{n}-T\right) P_{n} x \rightarrow 0$, so by Lemma 3: $\left\|\left(T_{n}-T\right) P_{n} P\right\| \rightarrow 0$. Now $\left(T-T_{n}\right) P_{n}(1-P)=\left(T-T_{n}\right) P_{n}\left(P_{n}-P\right)$ and $P_{n}-P=$ $-1 / 2 \pi i \int_{\Gamma}\left(R_{n}(z)-R(z)\right) d z=-1 / 2 \pi i \int_{\Gamma} R_{n}(z)\left(T-T_{n}\right) R(z) d z$, so there is a constant $C>0$ such that for $n$ large enough:

$$
\begin{aligned}
& \left\|\left(T_{n}-T\right) P_{n}(1-P)\right\| \leqslant C\left\|\int_{\Gamma}\left(T_{n}-T\right) P_{n} R_{n}(z)\left(T-T_{n}\right) R(z) d z\right\| \\
& \leqslant C\left(\left\|\int_{\Gamma}\left(T_{n}-T\right) P_{n} R_{n}(z) T_{n} R(z) d z\right\|+\left\|\int_{\Gamma}\left(T_{n}-T\right) P_{n} R_{n}(z) R(z) d z T\right\|\right) \\
& \leqslant C^{\prime}\left\|\left(T_{n}-T\right) T_{n}\right\|+C^{\prime \prime}\left\|\left(T_{n}-T\right) P_{n} \int_{\Gamma} R_{n}(z) R(z) d z T\right\|
\end{aligned}
$$

for some constants $C^{\prime}>0$ and $C^{\prime \prime}>0$. It remains to prove that

$$
\left\|\left(T_{n}-T\right) P_{n} \int_{\Gamma} R_{n}(z) R(z) d z T\right\| \rightarrow 0
$$

We define

$$
V_{n}=\int_{\Gamma} R_{n}(z) R(z) d z, \quad V=\int_{\Gamma} R(z)^{2} d z .
$$

For $n$ large enough, $V_{n} \in \mathscr{L}(X)$ and $\forall x \in X$

$$
\left\|\left(V_{n}-V\right) x\right\| \leqslant C \sup _{z \in \Gamma}\left\|\left(T_{n}-T\right) R(z)^{2} x\right\|
$$


with some constant $C>0$. Lemma 4 shows that $\forall x \in X, V_{n} x \rightarrow V x$ and Lemma 2 implies that $\forall x \in X,\left(T_{n}-T\right) P_{n} V_{n} x \rightarrow 0$. Finally, with Lemma 3 we conclude $\left\|\left(T_{n}-T\right) P_{n} V_{n} T\right\| \rightarrow 0$ and the proof is complete.

\section{A singularity subtraction example}

We consider the integral operator

$$
(T x)(t)=\int_{0}^{1} k(t, s) x(s) d s
$$

defined in the space $X=C[0,1]$ of complex valued continuous functions on $[0,1]$ normed by the uniform convergence norm.

The eigenproblem

$$
T x=\lambda x \quad x \neq 0
$$

may be rearranged in the form

$$
\int_{0}^{1} k(t, s)(x(s)-x(t)) d s+\int_{0}^{1} k(t, s) x(t) d t=\lambda x(t) .
$$

Let us approximate the first integral in the following way: First replace the kernel $k$ by an approximation $k_{n}$, where convergence holds in the sense of [2]; secondly, apply a quadrature rule with weights $\omega_{n j}$ and knots $t_{n j}$ satisfying the conditions specified in [2]. This leads to the problem

$$
\sum_{j=1}^{n} \omega_{n j} k_{n}\left(t, t_{n j}\right)\left(x\left(t_{n j}\right)-x(t)\right)+\int_{0}^{1} k(t, s) x(t) d s=\lambda x(t)
$$

which is the eigenproblem of the operator $T_{n} \in \mathscr{L}(X)$ defined by

$$
\left(T_{n} x\right)(t)=\sum_{j=1}^{n} \omega_{n j} k_{n}\left(t, t_{n j}\right)\left(x\left(t_{n j}\right)-x(t)\right)+\int_{0}^{1} k(t, s) x(t) d s .
$$

This operator is called the singularity subtraction approximation to $T$. As is proved in [2], under suitable conditions on $\left(k_{n}, \omega_{n j}, t_{n j}\right)$ operator $T_{n}$ satisfies $\mathrm{Cl}$ and $\mathrm{C} 2$.

The eigenvalues of $T_{n}$ may be computed by solving

$$
\left(T_{n} x\right)\left(t_{n i}\right)=\lambda_{n} x\left(t_{n i}\right), \quad i=1,2, \ldots, n,
$$

which leads to a matrix eigenproblem.

More precisely, we define the matrix $A_{n}=\left(a_{i j}\right)$ by

$$
a_{i j}= \begin{cases}\omega_{n j} k_{n}\left(t_{n i}, t_{n j}\right) & \text { if } j \neq i \\ \omega_{n i} k_{n}\left(t_{n i}, t_{n i}\right)-\varepsilon_{n}\left(t_{n i}\right) & \text { if } j=i\end{cases}
$$

where

$$
\varepsilon_{n}(t)=\sum_{j=1}^{n} \omega_{n j} k_{n}\left(t, t_{n j}\right)-\int_{0}^{1} k(t, s) d s
$$


If $u_{n} \in \mathbf{C}^{n}$ and $\lambda_{n} \in \mathbf{C}$ satisfy

$$
A_{n} u_{n}=\lambda_{n} u_{n}, \quad \max _{J}\left|u_{n j}\right|=1, \quad \lambda_{n} \neq 0,
$$

then $\lambda_{n}$ is an eigenvalue of $T_{n}$ and

$$
\phi_{n}(t)=\frac{\sum_{j=1}^{n} \omega_{n j} k_{n}\left(t, t_{n j}\right) u_{n j}}{\lambda_{n}+\varepsilon_{n}(t)} \quad(0 \leqslant t \leqslant 1)
$$

is an associated eigenfunction.

In the case of an eigenvalue $\lambda$ with algebraic multiplicity $m$ there will be, for $n$ large enough, a group of $m$ close eigenvalues $\lambda_{n, 1}, \lambda_{n, 2}, \ldots, \lambda_{n, m}$ (maybe some of them repeated) such that their arithmetic mean, say $\hat{\lambda}_{n}$, is an approximation to $\lambda$ of the order of $\left\|\left(T-T_{n}\right) P\right\|$ (see [3]).

Numerical computations are done with

$$
\begin{aligned}
k(t, s) & =\ln (1-\cos 2 \pi(t-s)) \quad t \neq s \\
k_{n}(t, s) & =\left\{\begin{array}{l}
k(t, s) \quad \text { if } \frac{1}{4 n} \leqslant|t-s| \leqslant 1-\frac{1}{4 n} \\
\ln \left(1-\cos \frac{\pi}{2 n}\right) \quad \text { otherwise }
\end{array}\right.
\end{aligned}
$$

and the trapezoidal rule as quadrature formula.

When $n=100$, matrix $A_{n}$ provides approximations of the order of $10^{-4}$ at nodal points $t_{n j}$ to the exact linearly independent eigenfunctions

$$
\begin{aligned}
& \phi(t)=-0.343778 \cos (6 \pi t-0.353927), \\
& \psi(t)=0.372441 \sin (6 \pi t-0.293559)
\end{aligned}
$$

associated with the exact eigenvalue $-1 / 3$ that, in turn, is approximated by -0.333356 , which is the arithmetic mean of two close eigenvalues of $A_{n}$ that differ by less than $10^{-5}$.

Table 3.1 shows relative errors of several eigenvalue approximations.

TABLE 3.1

\begin{tabular}{lccccc}
$\begin{array}{l}\text { Exact } \\
\text { Eigenvalue }\end{array}$ & Multiplicity & \multicolumn{3}{c}{ Approximate Eigenvalues and Relative Errors } \\
\hline & & \multicolumn{2}{c}{$n=10$} & \multicolumn{2}{c}{$n=100$} \\
\cline { 3 - 6 }$\lambda$ & $m$ & $\lambda_{n}$ & $\begin{array}{c}\text { Relative } \\
\text { Error }\end{array}$ & $\hat{\lambda}_{n}$ & $\begin{array}{l}\text { Relative } \\
\text { Error }\end{array}$ \\
\hline-1 & 2 & -0.994 & $0.60 \%$ & -1.000002 & $0.0002 \%$ \\
$-\ln 2$ & 1 & -0.684 & $1.32 \%$ & -0.693154 & $0.0010 \%$ \\
$-1 / 2$ & 2 & -0.505 & $1.00 \%$ & -0.500010 & $0.0020 \%$ \\
$-1 / 3$ & 2 & -0.357 & $7.10 \%$ & -0.333356 & $0.0068 \%$
\end{tabular}




\section{References}

[1] P. M. Anselone, Collectively Compact Operator Approximation Theory (Prentice-Hall, En glewood Cliffs, New Jersey, 1971).

[2] P. M. Anselone, "Singularity Subtraction in the Numerical Solution of Integral Equations", $J$. A ustral. Math. Soc. Ser. B 22 (1981), 408-418.

[3] F. Chatelin, Spectral Approximation of Linear Operators (Academic Press, New York, 1983). 